\title{
A CLUSTER BASED ENHANCEMENT TO AODV FOR INTER-VEHICULAR COMMUNICATION IN VANET
}

\author{
Aswathy $\mathrm{M} \mathrm{C}^{1}$ and Tripti $\mathrm{C}^{2}$ \\ ${ }^{1}$ Department of Computer Science \& Engineering, Rajagiri School of Engineering \& \\ Technology, Rajagiri valley, Cochin, India \\ aswathymcegmail.com \\ ${ }^{2}$ Department of Computer Science \& Engineering, Rajagiri School of Engineering \& \\ Technology, Rajagiri valley, Cochin, India \\ tripticerajagiritech.ac.in
}

\begin{abstract}
Vehicular Ad-hoc Networks (VANET) are a special kind of Mobile Ad-hoc network (MANET), in which vehicles on the road forms the nodes of the networks. Now a days, VANETs find several applications as an Intelligent Transportation System(ITS). Dynamic network architectures and node movement characteristics differentiates VANETs from other kinds of ad hoc networks. The dynamic change in topology shortens the effective time of routing. Hence routing in VANETs is complicated. Ad-hoc On-Demand Distance Vector $(A O D V)$ routing protocol is the most commonly used topology based routing protocol for VANET. During the route discovery process AODV broadcasts route request message (RREQ). It creates many unused routes between a source and a destination node. This paper aims at improving the performance of AODV by enhancing the existing protocol by creating stable clusters and performing routing by Cluster Heads and Gateway nodes.
\end{abstract}

\section{KEYWORDS}

VANET, AODV, Cluster

\section{INTRODUCTION}

In VANETs wireless-equipped vehicles form a network spontaneously while travelling along the road. Vehicles on the road can communicate with other vehicles on the road, called pure vehicle to vehicle $(\mathrm{V} 2 \mathrm{~V})$ communication, and vehicles can communicate with roadside equipments, called vehicle to infrastructure (V2I) communication. 


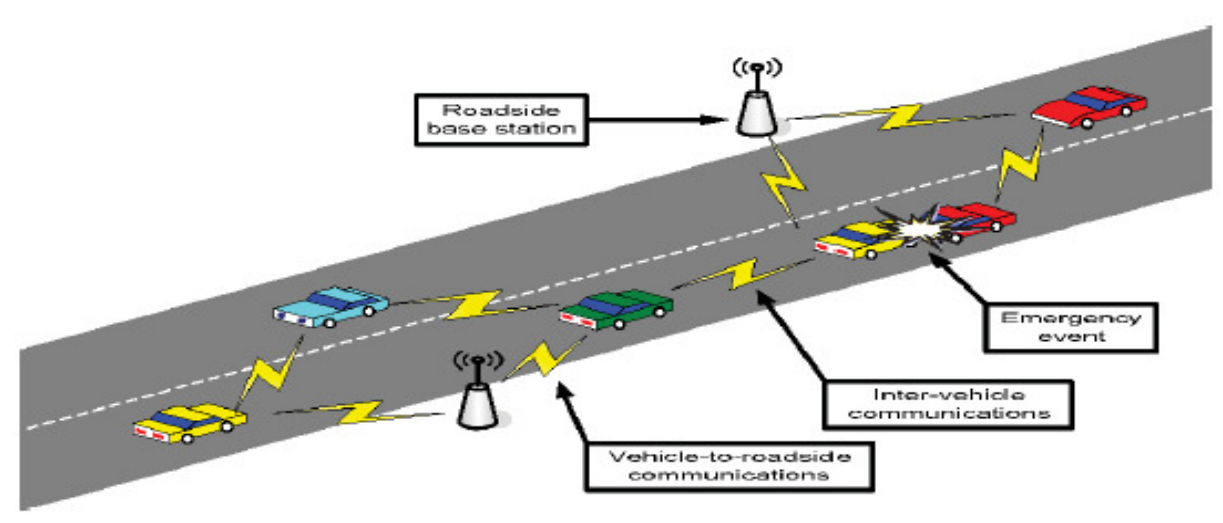

Figure 1. Basic architecture of VANET[13]

The node movement characteristics differentiate VANETs from other kinds of ad hoc networks. Therefore, the design of an efficient routing protocol for VANETs is very crucial. The bandwidth resources are limited in VANETs and also the topology of the network changes frequently. Hence, it is not necessary to maintain routes to each node. The dynamic change in topology shortens the effective time of routing. Also it reduces utilization rate of routing information. Therefore, on-demand routing protocols are good for VANETs.

On-demand routing protocols mainly involve two processes-Route Discovery and Route Maintenance. When a source node which has no routing information in the routing table needs to establish a route to the destination node, the route discovery process is activated. The source node broadcasts routing request packets across the network by flooding. When a route request packet reaches the destination node, the destination node sends a route response packet to the source node. This will set up a reverse path between the source node and the destination node. When the node changes, certain link on the activated path may break, then the route maintenance process will be started. Ad-hoc On-Demand Distance Vector (AODV) routing protocol is the most commonly used topology based routing protocol for VANET.

The remainder of the paper is structured as follows. Section II briefly introduces AODV and possible optimizations in AODV. Section III discusses the related works done on clustering and other improvements in AODV. Section IV introduces the proposed enhancement to AODV using Clusters. Section V describes the experimental setup for the implementation. Section VI concludes the paper and section VII introduces the future work.

\section{AODV AND OPTIMIZATION}

AODV is a reactive kind of protocol where the route from a source to a destination is created only when it is needed and it keeps these routes as long as they are desirable by the sources. AODV uses sequence numbers to ensure the freshness of routes and uses Hello messages to detect and monitor links to neighbours. Each active node periodically broadcasts a Hello message to all its neighbours. Since the Hello messages are periodically sent, when a node fails to receive several Hello messages from a neighbour, it detects a link failure. Every node of the network maintains a routing table which stores routing information.

\subsection{AODV CONTROL MESSAGES}

AODV defines three types of control messages for route discovery and maintenance, route request (RREQ) message, route reply (RREP) message and route error message (RREQ). 
International Journal of Grid Computing \& Applications (IJGCA) Vol.3, No.3, September 2012

\subsubsection{RREQ}

A source node which has no routing information to the destination in its routing table uses the route request message. Every RREQ carries a time to live (TTL) value that indicated the lifetime of the message, that is it states for how many hops this message should be forwarded. This value is set to a predefined value at the first transmission and increased at retransmissions. Retransmissions occur if no replies are received.

\subsubsection{RREP}

An intermediate node receiving the RREQ replies with a route reply message if it has a valid route to the requested address or it is the destination itself. The route reply message is unicasted back to the originator of a RREQ, this constructs a reverse path between the source and the destination.

\subsubsection{RERR}

A route error message is used when a node detects a link breakage in an active route. Each node keeps a precursor list, containing the IP address for each its neighbours that are likely to use it as a next hop towards each destination. When a link breakage in an active route is detected, a RERR message is used to notify other nodes of the loss of the link.

\subsection{AODV ROUTING PROCESS}

Routing using AODV is done by mainly two processes-Route Discovery and Route Maintenance.

\subsubsection{Route Discovery Process}

Route discovery process starts when a source node does not have routing information for a node to be communicated with. Route discovery is based on query, and the reply to this query is used to take a routing decision. Route discovery is initiated by broadcasting a RREQ message. If any of the neighbours has a route to the destination, it replies to the query with a route reply packet; otherwise, the neighbours rebroadcast the route query packet. Finally, some query packets reach to the destination. The route is established when a RREP message is received. A source node may receive multiple RREP messages with different routes. It then updates its routing entries if and only if the RREP has a greater sequence number, i.e. fresh information

\subsubsection{Route Maintenance Process}

Route maintenance process starts when link breakage happens. Since the nodes of the network are moving, link can break.If a node does not receive a HELLO message from one of its neighbours for specific amount of time called HELLO interval, then the node will detect a link breakage and the entry for that neighbour in the table will be set as invalid. The RERR message will be generated to inform other nodes of this link breakage .RRER messages inform all sources using a link when a failure occurs.

AODV can be optimized in many different ways. Channel Availability [10] can be used to enhance AODV for vehicle safety applications. Mobility parameters can be used while route discovery. Another method is to cluster the nodes of the network and managing routing of packets by cluster heads. The main idea behind clustering is to divide nodes of the network into multiple separate groups called clusters and forming a cluster structure. By clustering, the routing process can be focused to only a subset of nodes of the network, thus the routing task can be simplified. 
International Journal of Grid Computing \& Applications (IJGCA) Vol.3, No.3, September 2012

In a clustered network, instead of broadcasting the RREQ message, it can be sent to Cluster Heads. The Cluster Heads can find the routing information among the cluster members. If the route is available, the node sends a RREP message otherwise the RREQ is forwarded to other Cluster Heads via Gateway nodes. Thus the number of RREQ message for route discovery can be reduced considerably. This reduction in control messages can reduce the congestion in the network. Also overhead of the network to manage large number of packets can be reduced. Thus the performance of AODV can be improved.

\section{RELATED WORKS}

The AODV-Clustering [1] uses two route discovery mechanisms; Quick Route Discovery mechanism and the Traditional AODV Route Discovery mechanism. The protocol first uses the Quick Route Discovery mechanism. If a suitable route cannot be found, then it uses traditional AODV route discovery mechanism. When the algorithm uses the traditional AODV route discovery mechanism, it will flood the network with many control packets. In C-AODV[2], Clusters are formed based on the distance between the nodes and its cluster head. This is not suitable for VANETs since the distance between the nodes(vehicles) changes rapidly in VANETS. Enhanced AODV for directional flooding using Coordinate System [3] uses the concept of polar coordinate system. It limits the route discovery process to a limited region using GPS data like position, speed and track angle of source and destination node. Mobility-based Clustering in VANETs using Affinity Propagation [4] uses the idea of Affinity Propagation from a communications perspective, in a distributed manner. In the algorithm, each node in the network transmits the responsibility and availability messages to its neighbours, and then makes a decision on clustering independently. In Toward Strongly Connected Clustering Structure in Vehicular Ad hoc Networks [5], vehicles are assumed to use control channel to exchange periodic messages and gather information about their neighbourhood, and use one service channel to form the clusters and perform all intra-cluster communication tasks. Here the slowest vehicle among non-clustered neighbours initiates the cluster formation process. The algorithm avoids grouping vehicles whose relative velocity is greater than the threshold in one cluster. A Cluster-Based Directional Routing Protocol in VANET [14] proposes a cluster-based directional routing protocol (CBDRP) for highway scenarios, in which the header of a cluster selects another header according to the moving direction of vehicle to forward packets. In this protocol, it divides the vehicles with the same move direction to several clusters, then select one header in each cluster.

\section{ENHANCED AODV WITH CLUSTERING}

During the route discovery process AODV floods the entire network with large number of control packets, and hence it finds many unused routes between the source and destination [3]. This becomes a major drawback to AODV since this causes routing overhead, consuming bandwidth and node power.

The proposed enhancement to AODV optimizes AODV by reducing the number of control messages generated during the route discovery process. The optimization method uses the idea of clustering the nodes of the network and managing routing by cluster heads and gateway nodes. Routing using clusters effectively reduces the control messaged flooded during the route discovery process by replacing broadcasting of RREQ packets with forwarding of RREQ packets to Cluster Heads. Figure 2 shows the cluster formation in a VANET. 


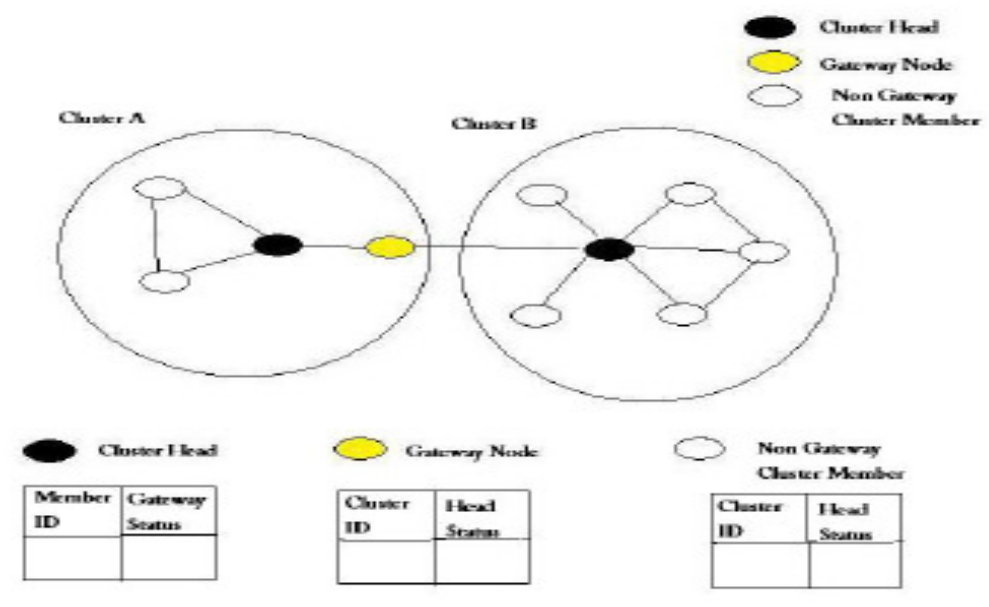

Figure 2. VANET Clusters with 10 nodes

The proposed algorithm for clustering in VANET can be done using three basic steps.

i. Cluster Formation

ii. Cluster Maintenance

iii. $\quad$ Clustered Routing

\subsection{Cluster Formation}

A cluster is characterised by a leader node called the Cluster Head node and a set of member nodes called the Cluster Members, having a single-hop link from the Cluster Head. A cluster has only one Cluster Head. The Cluster members may or may not be Gateways. Cluster Heads and Gateways are vital elements in routing.

In the proposed algorithm, to reduce the network overhead, cluster size is limited to single hop. Every node has a NODE-ID and a CLUSTER-ID. The CLUSTER-ID is the identified of the cluster of which the node is a member. Every node maintains a GATEWAY-TABLE which contains the CLUSTER-IDs of Cluster Heads, it can reach by single hop. A cluster head maintains a MEMBERS-TABLE to keep the list of members joined to this cluster. The number of cluster members is called its DEGREE.

Every node exchanges HELLO message to inform about their existence to its neighbour nodes and waits for CLUSTER_STATUS message from cluster head for a time period. The neighbouring Cluster Head will reply with its CLUSTER_STATUS message, which contains the Degree of the cluster. If the node gets more than one CLUSTER_STATUS message, it will update this information in the GATEWAY-TABLE and it will choose the cluster with highest degree to join. Then the node can send a request message JOIN_REQUEST to join the cluster, indicating whether it is a Gateway node or not. The Cluster Head will check its DEGREE. The node is allowed to join the cluster only if the DEGREE of the cluster is within the limits. This limits the number of Cluster Members and controls overhead of managing large number of Cluster Members by the Cluster Head. The cluster head sends a JOIN_ACCEPT message to the node if it can accept the request and updates the CLUSTER-MEMBERS table and the DEGREE. The requesting node sets the Head Status field in the GATEWAY-TABLE. If the node doesn't receive a CLUSTER_STATUS message within the time period, the node will elect itself as Cluster Head and updated its CLUSTER-ID with its NODE-ID. It will set its DEGREE as zero. 
International Journal of Grid Computing \& Applications (IJGCA) Vol.3, No.3, September 2012

\subsection{Cluster Maintenance}

Cluster maintenance is needed when there is a failure in an active link of the network. A link failure can be detected by the loss of HELLO messages from neighbours. If the cluster head doesn't receive HELLO message from its member within a time period, it will assume that the node is dead. The cluster head will delete the entry of the node from the members table. It also reduced its Degree by 1 . If a node doesn't receive CLUSTER_STATUS message from its Cluster Head within a time period, it will do the Cluster Join Procedure.

\subsection{Clustered Routing}

In Clustered AODV a source node seeking to send a data packet to a destination node checks its route table to see if it has a valid route to the destination node. If a route exists, it simply forwards the packets to the next hop along the way to the destination. On the other hand, if there is no route in the table, the source node begins a route discovery process. It sends a route request (RREQ) packet to its Cluster Head. The Cluster Head checks to see whether its member node has a route to the destination or the destination node itself. If it has a route, it will reply with a route reply (RREP) packet. If not, the Cluster Head will forward the RREQ packet to its Gateway Members. The Gateway members will forward the packet to Cluster Heads in its GATEWAY-TABLE. This process continues until the request reaches either an intermediate node with a route to the destination or the destination node itself. This route request packet contains the IP address of the source node, current sequence number, the IP address of the destination node, and the sequence number known last. An intermediate node will reply to the route request packet only if they have a destination sequence number that is greater than or equal to the number contained in the route request packet header. When an intermediate node forwards route request packet, it will record in its route table the address of the neighbour from which the first copy of the packet has come from. This recorded information is later used to construct the reverse path for the route reply (RREP) packet. When the route reply packet arrives from the destination or the intermediate node, the nodes forward it along the established reverse path and store the forward route entry in their route table.

The clustered routing can be summarised as:

- $\quad$ Source node sends RREQ to its Cluster Head.

- The Cluster Head checks whether Destination node is in its MEMBERS-TABLE.

- If so, it will forward the packet to the member

- Otherwise, it will forward packet to Gateway nodes in its MEMBERS-TABLE.

- When an intermediate node receives a RREQ packet, it checks whether it is the Destination node or is there any path to destination. If so, it will reply with an RREP packet

- When a Gateway node receives a RREQ packet, it will forward the packet to Cluster Heads in its GATEWAY-TABLE

\section{EXPERIMENTAL SETUP}

The proposed algorithm can be implemented in NS2.34. The mobility model for the VANET can be created using VanetMobiSim-1.1 and the communication can be defines in an NS2 TCL script. 


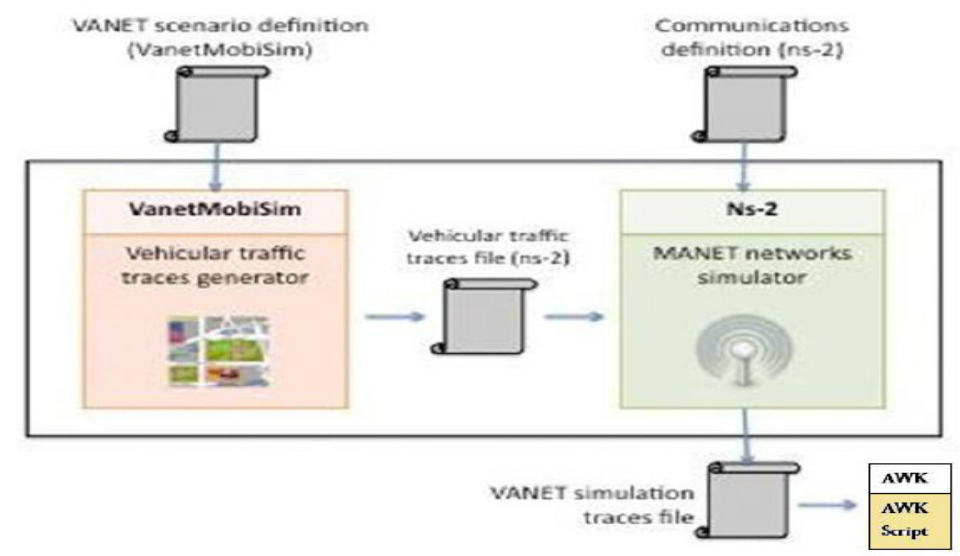

Figure 3. Interaction between Traffic and Network Simulators[15]

Both macro mobility features such as road topology, road structure such as number of lanes, single way or double-way movement etc, traffic light constraints, speed limits and micro mobility feature such as driving behaviour can be defined by using VanetMobisim. The output from VanetMobiSim simulation is a traffic generator trace file that corresponds to position coordinates of each vehicular node at every time steps. This traffic generated trace file is the mobility model serves as the input to NS2. To evaluate the performance of the network, the trace file generated from NS2 can be analysed and various performance parameters such as number of packets dropped, packet delivery ratio and end to end delay can be computed by using an AWK script.

\section{EXPECTED RESULTS}

The performance of the network can be analyzed by means of two parameters, throughput and percentage of packet loss over varying number of nodes in the network. Table 1 shows the simulation parameters used to evaluate the performance of AODV.

Table 1. SIMULATION PARAMETERS AND VALUES

\begin{tabular}{|l|l|}
\hline \multicolumn{1}{|c|}{ PARAMETERS } & \multicolumn{1}{c|}{ VALUES } \\
\hline Simulation Time & $3600 \mathrm{~s}$ \\
\hline Routing Protocol & AODV \\
\hline NS2 Version & 2.34 \\
\hline Transmission Range & $160 \mathrm{~m}$ \\
\hline MAC Layer Protocol & IEEE $802.11 \mathrm{p}$ \\
\hline Number of Nodes & $25,50,75$ \\
\hline Traffic Type & Constant Bit Rate(CBR) \\
\hline Packet Size & 512 bytes \\
\hline
\end{tabular}

Figure 4 and 5 show the throughput and percentage of packet loss of AODV for varying number of vehicles in the network. The expected result for AODVC is also given. 
International Journal of Grid Computing \& Applications (IJGCA) Vol.3, No.3, September 2012

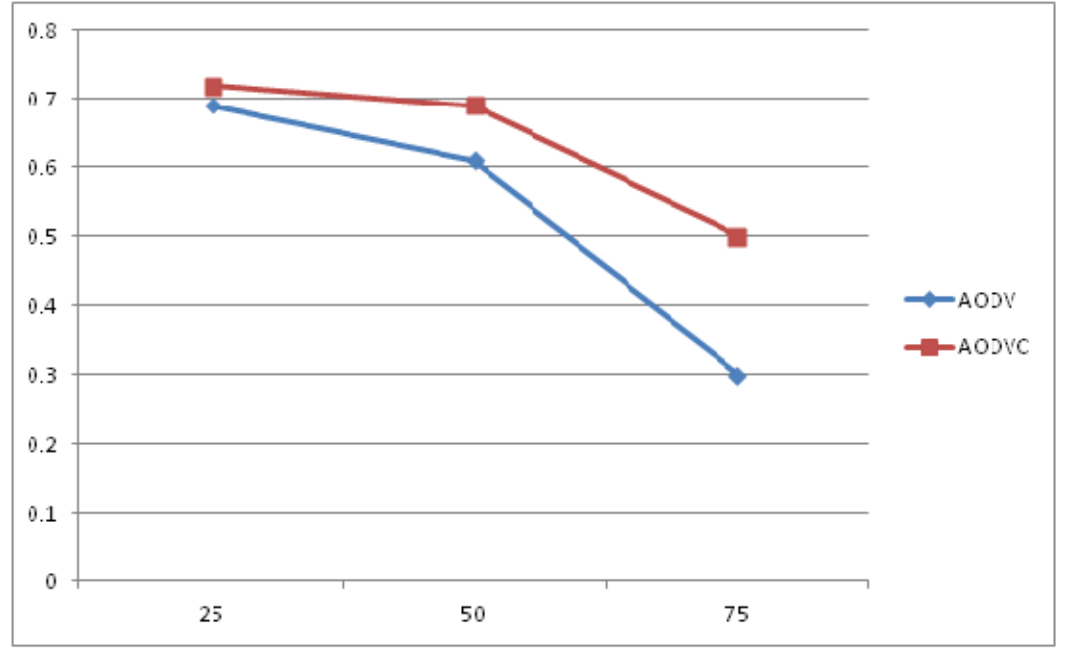

Figure 4. Throughput vs Number of nodes

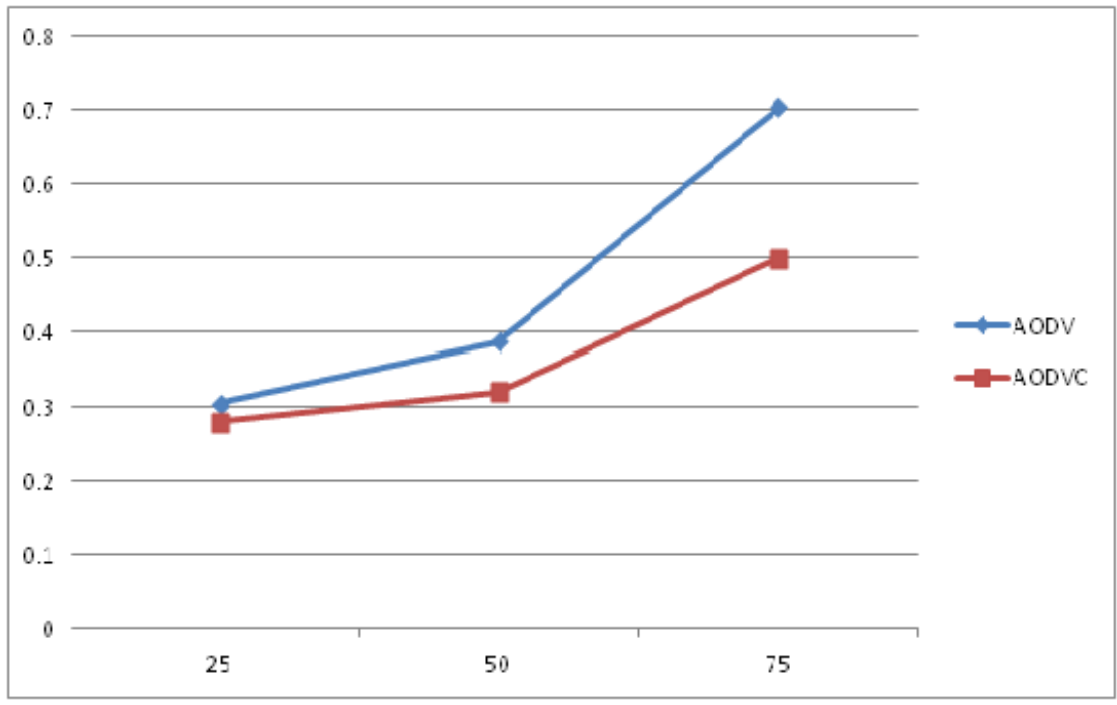

Figure 5. Percentage of packet loss vs Number of nodes

\section{CONCLUSIONS}

In the proposed method, the VANET is made into small clusters with long Cluster Head duration. The AODV protocol is optimized by replacing broadcasting of RREQ packets with forwarding of RREQ packets to Cluster Heads and there by managing routing by Cluster Heads and Gateway Nodes. This effectively reduces the total number of control packets generated during the route discovery process and also reduces the number of unused routes generated during the route discovery process. Thus the overhead of network in routing packets can be reduced and the efficiency of the protocol can be improved.

\section{FUTURE WORK}

The proposed scheme of clustering can be further enhanced by considering the mobility parameters of the vehicles such as direction, relative speed etc. When direction of the source and 
International Journal of Grid Computing \& Applications (IJGCA) Vol.3, No.3, September 2012

destinations nodes are taken into consideration, routing can be further refined to those nodes of the network which moves towards the direction of the destination. This will further reduce the unused routes generated during the route discovery process and thus will improve overall efficiency of the protocol.

\section{REFERENCES}

[1] ZHENG Kai, WANG Neng, LIU Ai-fang, "A new AODV based clustering routing protocol", International Conference on Wireless Communications, Networking and Mobile Computing, 2005 IEEE

[2] S.Thirumurugan, "C-AODV : Routing Protocol for Tunnel's Network", International Journal of Computer Sci ence and Technology, IJCST Vol. 2, Iss ue 1, March 2011

[3] S Reno Robert .R ,'Enhanced AODV for directional flooding using Coordinate System”, 2010 International Conference on Networking and Information Technology, 2010 IEEE

[4] Christine Shea, Behnam Hassanabadi and Shahrokh Valaee, "Mobility-based Clustering in VANETs using Affinity Propagation”, Global Telecommunications Conference, 2009. GLOBECOM 2009. IEEE

[5] Zaydoun Y. Rawshdeh, Syed Masud Mahmud ,'Toward Strongley Connected Clustering Structure in Vehicular Ad hoc Networks", Proceedings of the 2009 IEEE $70^{\text {th }}$ Vehicular Technology Conference:VTC2009-Fall, September 20-23, 2009,Anchorage,Alaska,USA

[6] Luciano Bononi , Marco Di Felice, "A Cross Layered MAC and Clustering Scheme for Efficient Broadcast in VANETs", International Conference on obile Adhoc and Sensor Systems,2007.MASS 2007. IEEE

[7] Yuyi Luo, Wei Zhang, Yangqing Hu “A New Cluster Based Routing Protocol for VANET”, 2010 Second International Conference on Networks Security, Wireless Communications and Trusted Computing, 2010 IEEE.

[8] N.MASLEKAR, M.BOUSSEDJRA, J.MOUZNA, H.LABIOD, “A Stable Clustering Algorithm for Efficiency Applications in VANETs", Wireless Communications and Mobile Computing Conference (IWCMC), 2011 IEEE.

[9] Venkata Manoj D, M. M. Manohara Pai, Radhika M.Pai, Joseph MOUZNA, "Traffic Monitoring and Routing in VANETs - A Cluster Based Approach”, 2011 11th International Conference on ITS Telecommunications, 2011 IEEE.

[10] Noppakun Yawan and Phongsak Keeratiwintakorn,'Efficiency Improvement of AODV for Vehicular Networks with Channel Availability Estimation", The 8th Electrical Engineeringl Electronics, Computer, Telecommunications and Information Technology (ECTI) Association of Thailand Conference 2011

[11] Peng Fan, James G. Haran, John Dillenburg, and Peter C. Nelson, "Cluster-Based Framework in Vehicular Ad-Hoc Networks", In Proceedings of ADHOC-NOW2005, LNCS 3738, pp. 32 - 42, Springer-Verlag Berlin Heidelberg 2005.

[12] Abolfazle Akbari, Mahdi Soruri, Seyed Vahid Jalali, "SURVEY OF STABLE CLUSTERING FOR MOBILE ADHOC NETWORKS”, 2009 Second International Conference on Machine Vision, 2010 IEEE

[13] Irshad Ahmed Soomro, Halabi Hasbullah,Jamalul-lail bin Ab Manan," User Requirements Model for Vehicular Ad hoc Network Applications", 20 I 0 IEEE

[14] Tao Song, Wei wei Xia, Tiecheng Song, Lianfeng Shen, "A Cluster-Based Directional Routing Protocol in VANET", 2010 IEEE

[15] Networking and Emerging Optimization, http://www.neo.lcc.uma.es/

[16] Sima, Ashwani Kush, "SIMULATION STUDY OF AODV\&DSR", International Journal of Computing and Business Research ISSN (Online), Volume 2 Issue 3 September 2011 
International Journal of Grid Computing \& Applications (IJGCA) Vol.3, No.3, September 2012

[17] Jia Junmin, Meng chun. A cluster-based peer-to-peer file sharing protocol for moblie ad hoc networks, I't International Symposium on Computer Network and Multimedia Technology (CNMT), 2009.

\section{Authors}

Aswathy M C: Did her B.Tech degree in Computer science and Engineering. She is now pursuing her post graduation in Computer Science and Engineering with specialization in Information Systems from Rajagiri School of Engineering and Technology, Kerala. Her areas of interest are Networking and Data structures. With 3 academic projects, and 1 publication to her credit, she wishes to continue in the field of research in networking.

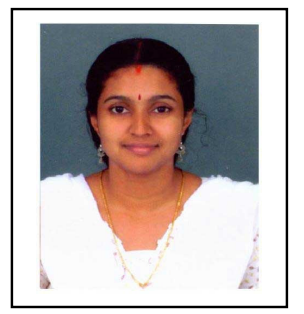

Tripti C: Is currently working as Asst.Professor in Rajagiri School of Engineering and Technology, Kerala. Did her M.Tech degree from C-DAC Noida and PG Diploma in Cyber law. She is a research scholar in the area of VANETs. Her areas of interest include Networking and Genetic Algorithms. With 5 academic projects, and 6 publications to her credit, she wishes to continue in

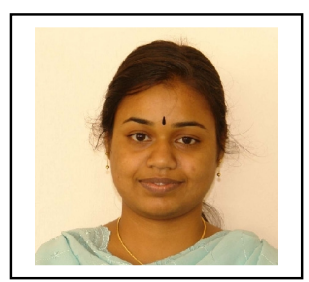
the field of research in VANETs. 